\title{
Review Article \\ Protective Effect of Tetramethylpyrazine on Myocardial Ischemia-Reperfusion Injury
}

\author{
Weidong Qian, ${ }^{1}$ Xingjiang Xiong, ${ }^{2}$ Zhuyuan Fang, ${ }^{3}$ Haiting Lu, ${ }^{1}$ and Zhensheng Wang ${ }^{1}$ \\ ${ }^{1}$ Department of Cardiology, Traditional Chinese Medicine Hospital of Wujin District, \\ Affiliated Hospital of Nanjing University of Chinese Medicine, Changzhou 213161, China \\ ${ }^{2}$ Department of Cardiology, Guang'anmen Hospital, China Academy of Chinese Medical Sciences, \\ Xicheng District, Beijing 100053, China \\ ${ }^{3}$ Department of Cardiology, Jiangsu Province Hospital of Traditional Chinese Medicine, Jiangsu 210029, China
}

Correspondence should be addressed to Xingjiang Xiong; 5administration@163.com and Zhuyuan Fang; jsfy@jsmail.com.cn

Received 6 February 2014; Revised 6 July 2014; Accepted 7 July 2014; Published 24 July 2014

Academic Editor: Bo Feng

Copyright (C) 2014 Weidong Qian et al. This is an open access article distributed under the Creative Commons Attribution License, which permits unrestricted use, distribution, and reproduction in any medium, provided the original work is properly cited.

Myocardial ischemia-reperfusion injury (MIRI) is a common pathological and physiological phenomenon. Tetramethylpyrazine is the extract of the traditional Chinese medicine Chuanxiong, which can exert protective effects on MIRI in multiple ways. This paper reviewed the current research progress and evidence about the cardiovascular effects of tetramethylpyrazine, which included protecting mitochondria and improving energy metabolism, scavenging oxygen free radicals (OFRs) to inhibit lipid peroxidation, attenuating calcium $\left(\mathrm{Ca}^{2+}\right)$ overload and maintaining $\mathrm{Ca}^{2+}$ homeostasis in cells, inhibiting apoptosis and protecting myocardial cells, interfering with the inflammatory reaction and mitigating cell injury, interfering with cell signaling pathways, and improving function of endothelial cells and protecting myocardial cells. However, further rigorously designed randomized controlled trials are warranted.

\section{Introduction}

Myocardial ischemia-reperfusion injury (MIRI) involves myocardial metabolic disorders and structural remodeling after reperfusion of the ischemic myocardium [1]. Previous studies have showed that the inflammatory response, platelet aggregation and microembolization, and cell death contributed significantly during the process of MIRI [2]. Conventional medicine therapy currently used in the treatment of MIRI includes nitrate, statins, $\mathrm{Ca}^{2+}$ antagonists, and angiotensin converting enzyme inhibitors (ACEI) [3]. However, undesirable effects of antianginal therapy do influence treatment adherence to a certain extent. A certain portion of patients with MIRI turned to traditional Chinese medicine therapy. Recent years have seen an increase in research relating to herbs for the treatment of MIRI, and tetramethylpyrazine (TMP) is among the most popular. TMP is an alkaloid found in the roots of Ligusticum chuanxiong Hort (LC; Umbelliferae) (as shown in Figures 1 and 2). TMP exerts a protective effect on MIRI in multiple ways with multiple targets, as described in this literature review.

\section{Pharmacology}

2.1. Protect Mitochondria and Improve Energy Metabolism. The heart requires a large amount of energy to maintain its normal physiological functions. Myocardial metabolic disorders have been reported to be involved in the pathogenesis of MIRI [4]. Myocardial ischemia reduces aerobic metabolism in the myocardium, and anaerobic metabolism becomes the main pathway. Anaerobic metabolism produces a large amount of acidic products, which in turn can induce intracellular acidic toxicity and thereby impair cell microstructure. Meanwhile, production of adenosine triphosphate (ATP) decreases rapidly, thereby reducing the mitochondrial activity of $\mathrm{Ca}^{2+}$-ATPase and $\mathrm{Mg}^{2+}$-ATPase. Therefore, mitochondrial $\mathrm{Ca}^{2+}$ levels are increased significantly. Disorders in 


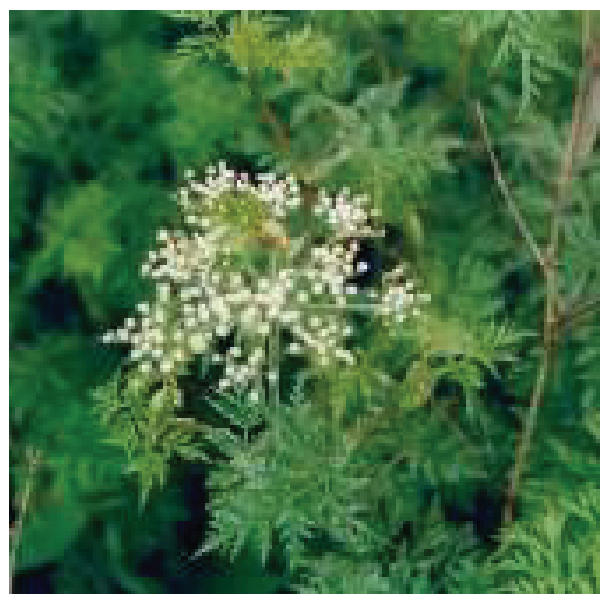

(a) Portion above ground

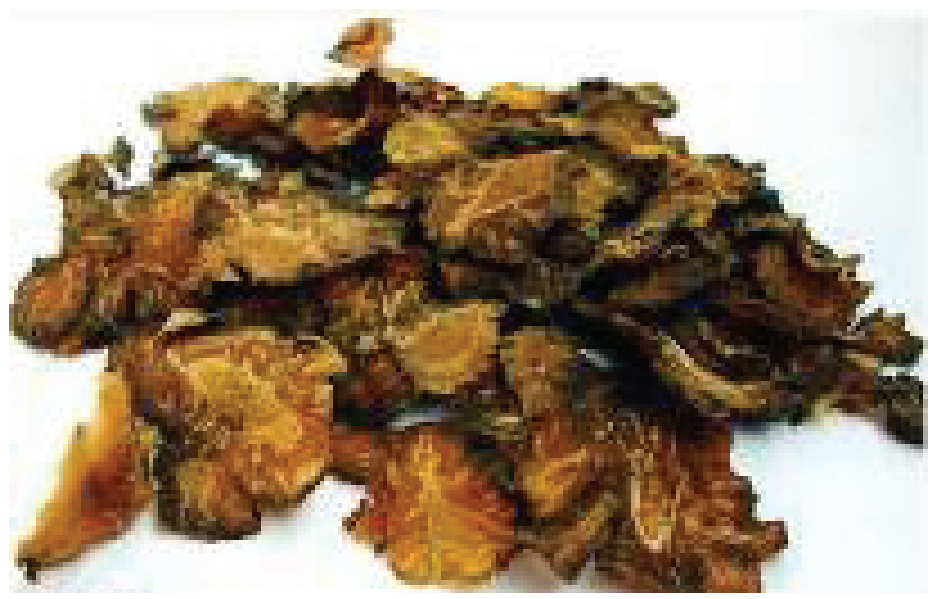

(b) Roots for pharmaceutical use

FIgURE 1: Morphology of tetramethylpyrazine.

TABLE 1: Protection of mitochondria and improvement of energy metabolism during MIRI.

\begin{tabular}{|c|c|c|}
\hline Pharmacological action & Methods & Reference \\
\hline \multirow{6}{*}{$\begin{array}{l}\text { Protect mitochondria } \\
\text { and improve energy } \\
\text { metabolism }\end{array}$} & $\begin{array}{l}\text { Reduce myocardial ATP } \\
\text { delay ATP breakdown }\end{array}$ & Nordlie et al. 2006 [5] \\
\hline & Protect $\mathrm{Na}^{+}-\mathrm{K}^{+}$-ATPase activity & Wang et al. 2003 [6] \\
\hline & Stimulate synthesis of protein and RNA & Zhu et al. 2010 [7] \\
\hline & Maintain the complete structure of biological membranes and myocardial fibers & Shi et al. $1998[8]$ \\
\hline & Reduce SDH and CCO deactivation & Wang et al. 1998 [9] \\
\hline & $\begin{array}{l}\text { Increase activity of } \mathrm{Ca}^{2+} \text {-ATPase and } \mathrm{Ca}^{2+}-\mathrm{Mg}^{2+} \text {-ATPase and regulate expression } \\
\text { of the } \mathrm{Bcl}-2 \text { gene }\end{array}$ & Wan et al. 2001 [10] \\
\hline
\end{tabular}<smiles>Cc1nc(C)c(C)nc1C</smiles>

FigURE 2: Molecular formula of tetramethylpyrazine.

energy metabolism can also induce mutations in myocardial genes and abnormal expression, thereby resulting in apoptosis [5].

Wang et al. reported that TMP can ameliorate MIRI by increasing energy production in myocardial cells [6]. A proposed mechanism is that TMP can reduce degradation of myocardial ATP and increase ATP generation. Through this pathway, energy storage in myocardial cells is increased, which could protect high-energy phosphate compounds in the myocardium.

Zhu et al. reported that $\mathrm{Na}^{+}-\mathrm{K}^{+}$-ATPase in myocardial tissues is not sensitive to ischemic injury but is sensitive to reperfusion injury [7]. TMP could protect the $\mathrm{Na}^{+}-\mathrm{K}^{+}$ATPase activity of ischemic myocardial tissues after reperfusion. Shi et al., using molecular biological methods, observed that TMP could increase absorption of ${ }^{3} \mathrm{H}$-leucine and ${ }^{3} \mathrm{H}$-uridine under oxygen- and sugar-deficient conditions in myocardial cells [8]. TMP could also stimulate the synthesis of protein and RNA as well as increase expression of nitric oxide synthase in oxygen- and sugar-deficient myocardial cells to enhance their tolerance of these deficiencies.

Wang and colleagues found that TMP could significantly alleviate or prevent the swelling or degeneration of mitochondria, breakage and dissolution of myofilaments, and the swelling and damage of the sarcolemma during MIRI [9]. Their study revealed that TMP could protect the myocardium by maintaining the complete structure of biological membranes and myocardial fibers and reducing injury to mitochondria.

Based on a study of key respiratory enzymes of mitochondria in vivo, Wan and colleagues reported that TMP strongly antagonizes the reduction of activity of succinate dehydrogenase $(\mathrm{SDH})$ and cytochrome oxidase (CCO) during MIRI [10]. $\mathrm{Li}$ et al. reported that the protective effect of TMP in a rat model of myocardial ischemic injury could be related to the increased activity of $\mathrm{Ca}^{2+}$-ATPase and $\mathrm{Ca}^{2+}-\mathrm{Mg}^{2+}$ ATPase and regulated expression of the Bcl-2 gene [11]. Wang et al. reported that TMP combined with L-arginine could improve mitochondrial function during MIRI by decreasing production of oxygen free radicals and reducing $\mathrm{Ca}^{2+}$ overload [12]. The effect of TMP on mitochondria protection and energy metabolism improvement during MIRI was shown in Table 1. 
2.2. Scavenge Oxygen Free Radicals (OFRs) to Inhibit Lipid Peroxidation. Free radicals are generated under physiological conditions to maintain normal metabolism. High levels of free radicals are harmful to the body, and so they are scavenged to maintain a dynamic balance. Superoxide dismutase (SOD), glutathione peroxidase (GSH-Px), heat-shock protein (HSP)70, and $\mathrm{H}_{2} \mathrm{O}_{2}$ are essential protective substances in the myocardium. Galang et al. demonstrated that treatment using SOD and $\mathrm{H}_{2} \mathrm{O}_{2}$ can significantly reduce the apoptosis of myocardial cells in rat hearts during MIRI in vitro [13]. Free radicals are among the key components of MIRI [14]. OFRs can injure biological membranes, proteins, nucleic acids, chromosomes, extracellular-space components, and mitochondria during MIRI and induce myocardial injury [15].

Studies have shown that TMP can scavenge reactive oxygen species, regulate production of nitric oxide (NO), and prevent the formation of peroxynitrites [16]. TMP can strongly scavenge OFRs and has effects upon cell toxicity [17]. Liu and colleagues suggested that the potential cardioprotective mechanism of TMP should contribute (at least in part) to its prominent antilipid peroxidation and antifree radical-formation effects. Hence, it could protect the heart from lipid peroxidation-induced toxicity [18]. Wang et al. reported that TMP can protect the myocardium by activating SOD and GSH-Px and stimulating HSP70 mRNA and the corresponding protein expression [19]. Chen and colleagues reported that TMP could suppress ischemiainduced ventricular arrhythmias and reduce the infarct size resulting from ischemia-reperfusion injury in vivo [20]. This cardioprotective effect of TMP may be associated with its antioxidant activity via induction of the expression of heme oxygenase- (HO-) 1 and its capacity for neutrophil inhibition. $\mathrm{Xu}$ et al. reported that in a rabbit model of MIRI, serum levels of malonaldehyde (MDA) in the TMP group were reduced significantly, whereas levels of GSH-Px and GSHPx lipid peroxide were increased considerably [21]. Therefore, TMP could inhibit OFR-induced damage to myocardial cells by protecting SOD activity, enhancing the scavenging of OFRs and reducing formation of lipid peroxides. Wan and colleagues reported that the activities of SOD, GSH-Px, CaATPase, and Na-K-ATPase in myocardial cell membranes were higher in the TMP-protected group than in the control group, whereas levels of MDA and $\mathrm{Ca}^{2+}$ were much lower [22]. Also, mitochondrial activities of SOD and GSH-Px were increased appreciably, whereas that of MDA was reduced severely.

Zhang and colleagues reported that TMP could significantly protect myocardial function in a rat model of MIRI. The proposed mechanism was that it could inhibit free-radical generation and scavenge OFRs [23]. Gu et al. reported that TMP postconditioning could significantly protect against MIRI in rats [24]. They found that TMP postconditioning could reduce the prevalence of arrhythmias and infarct size. They proposed that the mechanism could be related to increases in the production of SOD, NO, and nitric oxide synthase (NOS) and reduction of MDA generation. Wan and colleagues found that TMP has strong effects against the reduction of activity of SDH and cytochrome oxidation in MIRI. They proposed a mechanism related to increased scavenging of OFRs and inhibition of lipid peroxidation [25].

Piao et al. observed ischemia-reperfusion in rabbit hearts in vitro with TMP in a cardioplegic solution. They found that in the TMP group, MDA level was reduced and SOD activity increased significantly [26]. The microstructure of myocardial tissues was injured mildly, and they concluded that TMP had potent protective and antioxidant activities on the ischemic myocardium. Based on a model of myocardial cells with hypoxia-reoxygenation injury, Zhang et al. studied the influence of TMP on lactate dehydrogenase (LDH) in myocardial nutrient solution and MDA in myocardial cells. They found that levels of LDH and TMP were decreased by TMP [27]. Zhou and Liu reported that TMP has a strong protective effect towards hypoxia-reoxygenation injury in vitro that could be related to inhibition and scavenging of free radicals by TMP [28].

Chen and colleagues reported that TMP preconditioning could significantly improve left-heart function and reduce infarct size and arrhythmia in a rat model of MIRI in vitro. It could also increase the myocardial activity of SOD and GSH$\mathrm{Px}$, reduce MDA levels, upregulate expression of HSP70, and induce potent delays in protective functions [29]. Qin and colleagues also demonstrated that TMP could significantly antagonize the reduction of activity of SOD and GSH-Px and the increase in MDA content in a rat model of MIRI [30]. Yue et al., using a hemorrhagic-shock model of reperfusion in rabbits, found that serum levels of MDA were lowered dramatically and that the whole-blood activity of SOD and GSH-Px was significantly increased after TMP reperfusion [31]. The effect of TMP on scavenge OFRs to inhibit lipid peroxidation was shown in Table 2.

\subsection{Attenuate Calcium $\left(\mathrm{Ca}^{2+}\right)$ Overload and Maintain $\mathrm{Ca}^{2+}$} Homeostasis in Cells. $\mathrm{Ca}^{2+}$ is an endogenous messenger. Intracellular $\mathrm{Ca}^{2+}$ homeostasis is required to maintain and regulate cell function. Reperfusion can lead to disorders in intracellular $\mathrm{Ca}^{2+}$ and induce $\mathrm{Ca}^{2+}$ influx and disorders in the mechanisms for $\mathrm{Ca}^{2+}$ separation, which could induce myocardial $\mathrm{Ca}^{2+}$ overload. Increases in intracellular $\mathrm{Ca}^{2+}$ can activate endonuclease and lead to DNA breaks and apoptosis [32]. Therefore, reducing intracellular $\mathrm{Ca}^{2+}$ overload is crucial to protect the myocardium from MIRI.

It has been reported that TMP can significantly inhibit deactivation of $\mathrm{K}^{+}-\mathrm{Na}^{+}$-ATP and $\mathrm{Ca}^{2+}$-ATP in the membranes of myocardial cells during MIRI, which is important for the maintenance of intracellular $\mathrm{Ca}^{2+}$ stasis [33]. TMP can block the function of $\mathrm{Ca}^{2+}$ channels even more effectively than verapamil, and if it is combined with prostaglandin EI it can exert a strong synergistic protective effect in a rat model of MIRI [34-36]. TMP was found to not only block the entry of extracellular $\mathrm{Ca}^{2+}$ through $\mathrm{Ca}^{2+}$ channels but also inhibit the release of intracellular stored $\mathrm{Ca}^{2+}$ in vascular smooth muscle cells. TMP functioned as a true $\mathrm{Ca}^{2+}$ antagonist [37]. However, there are differences between in vitro and in vivo studies, and also results differ according to dose. More studies are required to obtain specificity of action [38]. 
TABLE 2: Scavenge OFRs to inhibit lipid peroxidation.

\begin{tabular}{lcr}
\hline Pharmacological action & Methods & Reference \\
& Prevent formation of peroxynitrites & Du et al. 2009 [15] \\
Scavenge OFRs to inhibit & Activate SOD and GSH-Px & Liu et al. 2005 [18] \\
lipid peroxidation & Induction of HO-1 & Wang et al. 2008 [19] \\
& Protect SOD activity & Chen et al. 2006 [20] \\
\hline
\end{tabular}

TABLE 3: Attenuate calcium overload and maintain cellular calcium homeostasis.

\begin{tabular}{lcc}
\hline Pharmacological action & Methods & Reference \\
\hline Attenuate $\mathrm{Ca}^{2+}$ overload and maintain & Reduce deactivation of $\mathrm{K}^{+}-\mathrm{Na}^{+}$-ATP and $\mathrm{Ca}^{2+}$-ATP & Ruiz-Meana and García-Dorado 2009 [32] \\
cellular $\mathrm{Ca}^{2+}$ homeostasis & Reduce level of Fas protein & Xu 2003 [38] \\
& Inhibition of early-after-depolarization & Zhang et al. 2009 [41] \\
\hline
\end{tabular}

Fas protein could increase intracellular $\mathrm{Ca}^{2+}$ overload. Li et al. reported that TMP could reduce Fas protein levels in the ischemic myocardium and moderate submicrostructure changes in myocardial cells [39]. They hypothesized that TMP has $\mathrm{Ca}^{2+}$ channel antagonist effects that, to some extent, protect the myocardium by reducing $\mathrm{Ca}^{2+}$ overload. However, illustration of the detailed mechanism requires further study.

$\mathrm{Xia}$ and $\mathrm{Wu}$ reported that TMP could potently protect $\mathrm{Ca}^{2+}$ transportation in isoproterenol-related myocardial ischemic injury [40]. Zhang et al. reported that low doses of TMP can reduce myocardial injury, increase the $\mathrm{Ca}^{2+}$ ATPase activity of myocardial mitochondria, improve cardiac function and intracellular $\mathrm{Ca}^{2+}$ concentrations in cardiocytes, and antagonize $\mathrm{Ca}^{2+}$ overload in rats with diastolic heart failure [41]. Zhou et al. reported that perfusion of $\mathrm{Ca}^{2+}$ and $\mathrm{Ca}^{2+}$ complexes in animal hearts could not induce similar myocardial injury to MIRI, especially early afterdepolarization (EAD) [42]. TMP could potently inhibit EAD, and this effect could be ameliorated by liquids containing high levels of $\mathrm{Ca}^{2+}$. It was considered that the effect of TMP in the treatment and prevention of MIRI was related to $\mathrm{Ca}^{2+}$ antagonism. The effect of TMP on attenuate calcium overload and maintain cellular calcium homeostasis was shown in Table 3 .

2.4. Inhibit Apoptosis and Protect Myocardial Cells. Apoptosis is programmed cell death. It may occur in certain pathophysiological conditions. It is a key step of myocardial cell death in acute myocardial ischemia-reperfusion [43], the population of which decides the severity of MIRI [44].

Duan and colleagues observed that apoptosis can occur in a rat model of MIRI and that cell populations as well as myocardial pathological changes are aggravated with time delay [45]. However, this could be alleviated by using TMP, which suggested that TMP inhibits apoptosis during MIRI. Xie et al. reported that TMP could not prevent but could slow down myocardial apoptosis induced in pressure-overloaded Sprague-Dawley rats, which suggests that TMP may have a cardioprotective effect [46].

Lipopolysaccharide (LPS) can boost the gene expression of cycloxygenase-2 (COX-2), which in turn can produce a series of inflammatory mediators. TMP can significantly inhibit expression of COX-2 mRNA and protein but does not affect COX-2 activity, which suggests that TMP inhibits COX-2 at the gene level by blocking LPS signals and antagonizing LPS-induced apoptosis of myocardial cells in rats [47].

Gene regulation of apoptosis involves promotion and inhibition. Bcl-2 is one of the most important apoptosis inhibitors, and Bax is a promoter. The $\mathrm{Bcl}-2 / \mathrm{Bax}$ ratio is considered to be a key factor of cell activity. Liu and colleagues illustrated that TMP could significantly upregulate bcl-2 expression without affecting Bax expression, which suggests that TMP can reduce MIRI-induced apoptosis [48]. This finding was confirmed by another study. TMP not only suppressed downregulation of expression of Bcl-2, upregulation of expression of Bax, and release of mitochondrial cytochrome $\mathrm{c}$ to the cytosol, but also attenuated caspase3 activation and eventually protected against $\mathrm{H}_{2} \mathrm{O}_{2}$-induced apoptosis [49]. Zhang and colleagues suggested that myocardial apoptosis and the Fas/FasL system are involved in the occurrence and development of myocardial ischemic injury in rats [50]. Zhang and colleagues stated that TMP may inhibit Fas/Fas L and caspase-3 levels in ischemic myocardial reperfusion and that caspase- 3 is one of the factors of apoptosis [51]. That study also revealed that the Fas death receptor pathway participates in MIRI by which TMP inhibits apoptosis.

Activated STAT3 can be transmitted inside a cell and, if combined with specific DNA series, can upregulate $\mathrm{Bcl}-2$ expression, thereby promoting the transcription of inducible NOS, COX-2, and manganese superoxide dismutase and inhibiting apoptosis of myocardial cells. Zhai and colleagues reported that the JAK2/STAT3 signaling pathway is involved in relieving MIRI in rats using TMP [52].

Zhao and colleagues observed the influence of ligustrazine ferulate (LF) postconditioning in a rat model of MIRI. They found that TMP and LF could accelerate the heart rate, increase left-ventricular pressure of the late systolic period, increase the rate $(\mathrm{dP} / \mathrm{dt} \max )$ of left-ventricular pressure rise in early systole, reduce the left-ventricular pressure of the late diastolic period, increase serum SOD activity, and reduce MDA content. These phenomena reduced myocardial infarct size, the apoptosis index and expression of Fas protein [53]. 
TABLE 4: Inhibition of apoptosis.

\begin{tabular}{lrr}
\hline Pharmacological action & Methods & Reference \\
\hline & Stop transmission of LPS signal et al. 2004 [46] \\
Inhibit apoptosis & Promote bcl-2 expression, increase the Bcl-2/Bax ratio & Wan et al. 2004 [47] \\
& Attenuate activation of caspase-3 & Liu and Niu 2011 [48] \\
& Inhibit the intensity of Fas/Fas L & Zhang et al. 2009 [50] \\
& Activate the JAK2/STAT3 signal pathway & Zhang et al. 2007 [51] \\
\hline
\end{tabular}

TABLE 5: Interference with the inflammatory reaction.

\begin{tabular}{lcc}
\hline Pharmacological action & Methods & Reference \\
\hline & Decrease activity of p38MAPK & Castaneda et al. 2003 [58] \\
Interference with the inflammatory reaction & Inhibit the expression of E-selectin and P-selectin & Shang et al. 2008 [59] \\
& Inhibit ICAM-1 expression & Pan and Jin 2008 [60] \\
\hline
\end{tabular}

They believe that LF postconditioning can relieve MIRI in rats in vivo. The effect of TMP on inhibition of apoptosis was shown in Table 4.

\subsection{Interfere with the Inflammatory Reaction and Mitigate Cell} Injury. Recently, the relationship between the inflammatory reaction and MIRI has become well-established [54]. The influence of TMP on decreasing cerebral ischemia and reperfusion-induced effects on activation of production of inflammatory cells and proinflammatory mediators has been confirmed [55]. Inflammatory injury continues throughout MIRI and is activated in the ischemic period and obviously aggravated upon reperfusion [56]. Interference with neutrophils and proinflammatory factors could mean an improvement in MIRI.

$\mathrm{Hu}$ and colleagues found that the activity of SOD and GSH-Px in the TMP preconditioning group was promoted considerably, whereas that of $\mathrm{MDA}, \mathrm{LDH}$, creatinine kinase (CK), tumor necrosis factor- (TNF-) $\alpha$, and interleukin- (IL) 6 was decreased and myocardial infarct size reduced as the ST interval declined [57]. These protections are due to the increased activity of SOD, GSH-Px, and the inhibited inflammatory reaction. In MIRI, high levels of free radicals activates p38 mitogen activated protein kinase (p38MAPK) and nuclear factor kappa-light-chain-enhancer of activated B cells (NF-B), both of which induce the generation of TNF- $\alpha$ and IL-6 [58]. Shang et al. reported that TMP could decrease p38MAPK activity, inhibit the expression of TNF- $\alpha$ and IL- 6 , and thereby protect the myocardium [59].

Pan and Jin reported that TMP combined with LF may help to protect against MIRI in rats by reducing serum CK levels as well as inhibiting expression of E-selectin and Pselectin of endothelial cell adhesion molecules [60]. Yang and colleagues reported that TMP, LF, and their combination can alleviate MIRI through inhibition of expression of the endothelial cell adhesion molecule mRNA of E-selectin, Pselectin, and intercellular adhesion molecule- (ICAM-) 1, among which the combination group was the most prominent [61]. The effect of TMP on inhibition of inflammatory reaction was shown in Table 5.
2.6. Interfere with Cell Signaling Pathways. The reperfusion injury salvage kinase (RISK) signaling pathway is a type of signal-regulated kinase which includes phosphatidylinositol3 kinase (PI3K)/protein kinase B (Akt), extracellular signalregulated kinase $(E R K 1 / 2)$, protein kinase $C(P K C)$, and protein kinase $\mathrm{G}$ (PKG). Interference of the RISK signaling pathway (to elicit relief from MIRI) has become a "hot" research target in recent years [62].

Phosphorylation of endothelial nitric oxide synthase (eNOS) is one of the downstream targets in the PI3K/Akt pathway [63]. As reported by Lv et al., TMP has antiapoptotic and cardioprotective effects against MIRI and acts through the PI3K/Akt pathway [64]. In addition, phosphorylation of eNOS with subsequent production of NO was found to be an important downstream effector contributing significantly to the cardioprotective effect of TMP.

In vivo and cell-level research has shown that PKC and $\mathrm{Gi} /$ o protein are involved in the protection of preconditioned myocardial cells. Liang et al. reported that PKC blockers and $\mathrm{Gi} / \mathrm{o}$ protein deactivators could abrogate the protection of hypoxic preconditioning and TMP preconditioning, which suggested that the mechanism of TMP preconditioning protection was probably related to $\mathrm{PKC}$ and Gi/o protein [65]. Chen and colleagues reported that TMP could induce delayed cardioprotective effects by activation of PKC and extracellular signal-regulated protein kinase 1/2 signaling pathways as well as subsequent increased expression of HSP70 in rat neonatal cardiomyocytes [66].

Adiponectin acts on AdipoR1 to activate the AMPK channel to promote energy utilization in infarcted areas, maintain ATP levels, and inhibit apoptosis. C. Q. Li and Y. X. $\mathrm{Li}$ reported that the protection afforded by TMP in MIRI rats could be related to increased levels of adiponectin [67]. The effect of TMP on interference with cell signaling pathways was shown in Table 6.

2.7. Improve Function of Endothelial Cells and Protect Myocardial Cells. Ischemic reperfusion in MIRI leads to disorder in endothelial cells. Wang et al. reported that TMP could protect coronary endothelial cells, increase the NO level, and reduce the level of endothelial cells in humans to alleviate MIRI [68]. 
TABLE 6: Interference with cell signaling pathways.

\begin{tabular}{lcr}
\hline Pharmacological action & Methods & Reference \\
\hline & Activate the PI3K/Akt-eNOS & Fulton et al. 1999 [63] \\
Interfere cell signal pathway & Increase the activity & of PKC and Gi/o \\
& Increase expression of HSP70 et al. 2012 [64] & Liang et al. 2001 [65] \\
& Increase adiponectin level & Chen et al. 2007 [66] \\
\hline
\end{tabular}

TABLE 7: Improve function of endothelial cells.

\begin{tabular}{lcc}
\hline Pharmacological action & Methods & Reference \\
\hline \multirow{2}{*}{$\begin{array}{l}\text { Improve endothelial cell function } \\
\text { Increase the level of NO and decrease the number of endothelial cells }\end{array}$} & C. Q. Li and Y. X. Li 2010 [67] \\
& Increase the level of 6-Keto-PGF $_{1 \alpha}$ and decrease the level of TXB T $_{2}$ & Wang et al. 2001 [68] \\
\hline
\end{tabular}

Liang and colleagues reported that ischemic preconditioning reduces levels of endothelial cells and TTXB2 after reperfusion, while that of 6-Keto-PGF ${ }_{1 \alpha}$ is increased [69]. This phenomenon could explain how preconditioning can stimulate endothelial cells to release more vasodilator substances during ischemic reperfusion to protect themselves. Those studies illustrated that TMP preconditioning could protect against injury to vascular endothelial cells due to ischemic reperfusion. Li et al. reported that the antiapoptotic effect of TMP and salvianolic acid B on rheologically induced injury to endothelial cells was likely to contribute to their efficacy [70]. The effect of TMP on improving function of endothelial cells was shown in Table 7.

\section{Discussion and Prospects}

In recent years, research into the treatment and prevention of MIRI with TMP has been fruitful. The common view is that TMP protects against MIRI by multiple mechanisms: scavenging OFRs, attenuating $\mathrm{Ca}^{2+}$ overload, protecting endothelial cells, inhibiting apoptosis, and affecting cytokine expression [71].

However, there are so many drawbacks in this research. The lack of multicenter, randomized, double-blind, longterm, and large-scale clinical trials, the differentiation of dose, evaluation standards, and the influence of complex agents affect the evaluation of TMP [72]. Current research remains at a simple, repeatable stage with very few insights into signalrelated pathways, gene regulation, and receptor channels; more information is needed to elucidate the mechanisms involved [73]. As a key factor to determine the severity of reperfusion injury, the mitochondrial permeability transition pore is becoming a new important target in cardioprotection research. If TMP is a specific $\mathrm{Ca}^{2+}$ antagonist, then why does it exert different effects in vivo and in vitro and in different doses? Is the antagonism related to the severity of illness? These problems should be researched further [38]. $\mathrm{K}^{+}$channels (especially the $\mathrm{K}_{\mathrm{ATP}}$ channel) are closely related to the onset, development, and prevention of myocardial ischemia. $\mathrm{K}_{\mathrm{ATP}}$ channel openers have been attracting much attention but have been mostly ignored by TMP researchers.
MIRI is a complicated pathophysiological process with multiple synergistic actions, and its mechanism of action has yet to be elucidated. Under the direction of traditional Chinese medicine and advanced scientific methodology, the mechanism by which TMP treats and prevents MIRI should be researched further.
Abbreviations
COX-2: Cyclooxygenase-2
eNOS: $\quad$ Endothelial nitric oxide synthase
ERK1/2: Extracellular signal-regulated kinases
GSH-Px: Glutathione peroxidase
HO-1: Heme oxygenase-1
HSP70: $\quad$ Heat shock protein 70
ICAM-1: Intercellular adhesion molecule-1
LDH: $\quad$ Lactate dehydrogenase
LF: $\quad$ Tetramethylpyrazine ferulate
LPS: $\quad$ Lipopolysaccharide
MIRI: Myocardial ischemia-reperfusion injury
NF- $\kappa \mathrm{B}$ : Nuclear factor
kappa-light-chain-enhancer of activated B cells
OFR: $\quad$ Oxygen free radical
p38MAPK: p38 mitogen-activated protein kinase
RISK: Reperfusion injury salvage kinase
PI3K: $\quad$ Phosphatidylinositol-3 kinase
SDH: $\quad$ Succinate dehydrogenase
SOD: $\quad$ Superoxide dismutase
TMP: $\quad$ Tetramethylpyrazine.

\section{Conflict of Interests}

All authors declare that they have no conflict of interests.

\section{Acknowledgments}

The current work was supported in part by the National Natural Science Foundation Project of China (no. 81273713) and Jiangsu Provincial Administration of traditional Chinese Medicine (no. LZ13142). 


\section{References}

[1] L. C. Becker and G. Ambrosio, "Myocardial consequences of reperfusion," Progress in Cardiovascular Diseases, vol. 30, no. 1, pp. 23-44, 1987.

[2] A. T. Turer and J. A. Hill, "Pathogenesis of myocardial ischemiareperfusion injury and rationale for therapy," The American Journal of Cardiology, vol. 106, no. 3, pp. 360-368, 2010.

[3] D. S. Villanueva, P. Poirier, P. R. Standley, and T. L. Broderick, "Prevention of ischemic heart failure by exercise in spontaneously diabetic BB Wor rats subjected to insulin withdrawal," Metabolism, vol. 52, no. 6, pp. 791-797, 2003.

[4] G. Lopaschuk, "Regulation of carbohydrate metabolism in ischemia and reperfusion," American Heart Journal, vol. 139, no. 2, pp. S115-S119, 2000.

[5] M. A. Nordlie, L. E. Wold, B. Z. Simkhovich, C. Sesti, and R. A. Kloner, "Molecular aspects of ischemic heart disease: ischemia/reperfusion-induced genetic changes and potential applications of gene and RNA interference therapy," Journal of Cardiovascular Pharmacology and Therapeutics, vol. 11, no. 1, pp. 17-30, 2006.

[6] W. T. Wang, Z. J. Xu, L. N. Lin et al., "Effect of ligustrazine on myocardial cell energy metabolism in the reperfusion injury after myocardial ischemia in rabbits," Chinese Journal of Critical Care Medicine, vol. 23, no. 3, pp. 129-130, 2003.

[7] H. B. Zhu, M. M. Wang, and B. Zhang, "Effect of myocardial ischemia reperfusion in rabbits on $\mathrm{Na}^{+}-\mathrm{K}^{+}$-ATPase and protective effect of ligustrazine pretreatment," Hubei Journal of Traditional Chinese Medicine, vol. 32, no. 8, pp. 5-6, 2010.

[8] D. Z. Shi, K. J. Chen, and P. Zhong, "The effects of ligustrazine on synthesis of protein and RNA, and NOS gene expression in cultural myocardial cells with hypoxia and lack of sugar," Chinese Pharmaceutical Journal, vol. 33, no. 12, pp. 724-726, 1998.

[9] Z. M. Wang, Z. J. Xu, and W. T. Wang, "Ultrastructural changes of myocardial ischemia reperfusion injury in rabbits and protective effects of ligustrazine," Journal of Wenzhou Medical College, vol. 28, no. 1, pp. 15-17, 1998.

[10] F. S. Wan, B. Liu, X. M. Zhao et al., "Effects of ligustrazine on respiratory enzymes inthe myocardial mitochondria of the ischemia-reperfusion rats," Chinese Journal of Pathophysiology, vol. 17, no. 1, p. 58, 2001.

[11] Y. Li, F. S. Wan, Y. F. Wan et al., "Protective effects of ligustrazine against myocardial ischemia injury of rat," Chinese Traditional Patent Medicine, vol. 25, no. 8, pp. 646-648, 2003.

[12] W. T. Wang, F. Y. Wang, W. Wang et al., "Effect of ligustrazine and L-arginine on function of mitochondria in myocardial cell in the reperfusion injury after myocardial ischemia in rabbits," Chinese Journal of Arteriosclerosis, vol. 14, no. 11, p. 955, 2006.

[13] N. Galang, H. Sasaki, and N. Maulik, "Apoptotic cell death during ischemia/reperfusion and its attenuation by antioxidant therapy," Toxicology, vol. 148, no. 2-3, pp. 111-118, 2000.

[14] A. A. Wolff, H. H. Rotmensch, W. C. Stanley, and R. Ferrari, "Metabolic approaches to the treatment of ischemic heart disease: the clinicians' perspective," Heart Failure Reviews, vol. 7, no. 2, pp. 187-203, 2002.

[15] J. Du, C. J. Wang, and Z. M. Jing, "Study on damage of calcium overload and the role of oxygen free radical mechanism of myocardial ischemia reperfusion," Health Vocational Education, vol. 27, no. 11, pp. 121-123, 2009.

[16] Z. Zhang, T. Wei, J. Hou, G. Li, S. Yu, and W. Xin, “Tetramethylpyrazine scavenges superoxide anion and decreases nitric oxide production in human polymorphonuclear leukocytes," Life Sciences, vol. 72, no. 22, pp. 2465-2472, 2003.

[17] Z. H. Zhang, S. Z. Yu, Z. T. Wang et al., "Scavenging effects of tetramethylpyrazine on active oxygen free radicals," Acta Pharmacologica Sinica, vol. 15, no. 3, pp. 229-231, 1994.

[18] C. F. Liu, C. H. Lin, C. F. Chen, T. Huang, and S. Lin, "Antioxidative effects of tetramethylpyrazine on acute ethanolinduced lipid peroxidation," The American Journal of Chinese Medicine, vol. 33, no. 6, pp. 981-988, 2005.

[19] F. Wang, L. Z. Shang, J. R. Wang et al., "Effect and mechanism of chuanxiongzine pretreatment on rats myocardial ischemia reperfusion injury," Henan Journal of Traditional Chinese Medicine, vol. 28, no. 4, pp. 26-28, 2008.

[20] S.Y. Chen, G. Hsiao, H. R. Hwang, P. Cheng, and Y. Lee, "Tetramethylpyrazine induces heme oxygenase-1 expression and attenuates myocardial ischemia/reperfusion injury in rats," Journal of Biomedical Science, vol. 13, no. 5, pp. 731-740, 2006.

[21] Z. J. Xu, W. T. Wang, and D. Li, "Protective effects of ligustrazine on myocardial ischemia reperfusion injury in rabbits and its mechanism," Basic Medical Science and Clinics, vol. 17, no. 4, pp. 68-71, 1997.

[22] F. S. Wan, X. M. Zhao, B. Liu et al., "Protective effects of ligustrazine on myocardial ischemia reperfusion injury in rats," Journal of Clinical Pharmacology and Therapeutics, vol. 3, no. 3, pp. 184-186, 1998.

[23] Q. F. Zhang, L. J. Chen, and M. Li, "Protective effects of ligustrazine on myocardial ischemia reperfusion injury in rats," China Medical Herald, vol. 6, no. 9, pp. 17-18, 2009.

[24] Y. C. Gu, X. L. Yu, and W. J. Guo, "Protective effect of tetramethylpyrazine postconditioning on ischemia reperfusion injured myocardium in rats," International Journal of Cardiovascular Disease, vol. 36, no. 5, pp. 304-307, 2009.

[25] F. S. Wan, B. Liu, X. M. Zhao et al., "Effects of ligustrazine on respiratory enzymes in the myocardial mitochondria of the ischemia reperfusion rats," Chinese Journal of Pathophysiology, vol. 17, no. 1, pp. 58-60, 2001.

[26] Z. G. Piao, H. S. Piao, G. C. Jin et al., "An experimental study of ligustrazine on ischemic myocardial protection and scavenging oxygen free radical," Chinese Wild Plant Resources, vol. 19, no. 5, pp. 46-48, 2000.

[27] X. X. Zhang, J. P. Zhang, and L. J. Chen, "To study the protective effect of ligustrazine on myocardial cell injury," Chinese Community Doctors, vol. 11, no. 18, article 3, 2009.

[28] Y. P. Zhou and Q. Liu, "Protective effects of ligustrazine on hypoxia/reoxygenation injury in cardiomyocytes," Practical Clinical Medicine, vol. 12, no. 2, pp. 5-7, 2011.

[29] D. Chen, D. Liu, G. H. Zeng et al., "HSP70 mediated delayed protective effect of ligustrazine preconditioning on isolated rat heart," Pharmacology and Clinics of Chinese Material Medica, vol. 23, no. 5, pp. 80-83, 2007.

[30] X. C. Qin, G. Q. Fen, R. F. Fu et al., "Experimental study on the protective effect of ligustrazine on myocardialischemia reperfusion injury in rats," Henan Journal of Traditional Chinese Medicine, vol. 17, no. 5, pp. 274-276, 1997.

[31] Y. L. Yue, H. M. Li, Z. F. Zhao et al., "Protective effects of ligustrazine on hemorrhagic shock and reperfusion injury in rabbits," Journal of Xi'An Medical University, vol. 19, no. 1, pp. 4-7, 1998.

[32] M. Ruiz-Meana and D. García-Dorado, "Pathophysiology of ischemia reperfusion injury: new therapeutic options for acute myocardial infarction," Revista Espanola de Cardiologia, vol. 62, no. 2, pp. 199-209, 2009. 
[33] M. R. Siegfried, J. Erhardt, T. Rider, X.-. Ma, and A. M. Lefer, "Cardioprotection and attenuation of endothelial dysfunction by organic nitric oxide donors in myocardial ischemiareperfusion," The Journal of Pharmacology and Experimental Therapeutics, vol. 260, no. 2, pp. 668-675, 1992.

[34] G. S. Zhao, H. J. Yang, Z. Y. Zhou et al., "To study the effect of ligustrazine reperfusion effect," Chinese Emergency Medicine, vol. 6, no. 5, pp. 222-224, 1997.

[35] G. S. Zhao, H. J. Yang, B. H. Zuo et al., "Comparison of protective effects of ligustrazine and danshen on oxygen paradox in isolated rat hearts," Tianjin Medical Journal, vol. 24, no. 5, pp. 289-292, 1996.

[36] F. Run-Fang, F. Guo-Qing, L. Jie, F. Guo-Yi, and W. Shi-Ai, "Protective effect of prostaglandin E1 combined with ligustrazine on myocardial ischemia reperfusion injury in rats," Chinese Pharmacological Bulletin, vol. 14, no. 6, pp. 548-550, 1998.

[37] P. K. T. Pang, J. J. Shan, and K. W. Chiu, "Tetramethylpyrazine, a calcium antagonist," Planta Medica, vol. 62, no. 5, pp. 431-435, 1996.

[38] H. Xu, "Ligustrazine cardiovascular pharmacology and calcium antagonism," Chinese Journal of Integrated Traditional and Western Medicine, vol. 23, no. 5, pp. 376-377, 2003.

[39] Y. Li, T. C. Wang, W. Q. Gong et al., "Effects of ligustrazine on Fas protein expression of myocardial ischemia reperfusion in rats," Journal of Fourth Military Medical University, vol. 20, no. 6, pp. 515-518, 1999.

[40] L. M. Xia and J. X. Wu, "Effects of ligustrazine on dysfunction of myocardial nuclear calcium transport," Chinese Journal of Clinical Pharmacology and Therapeutics, vol. 7, no. 2, pp. 124126, 2002.

[41] X. Zhang, W. Liu, J. Zhou, and C. Fan, "Studies on protection and mechanism of tetramethylpyrazine on myocardial injury of rats with DHF," China Journal of Chinese Materia Medica, vol. 34, no. 21, pp. 2808-2812, 2009.

[42] C. Zhou, Y. Liu, L. Guan, and H. Wang, "Inhibitory effects of tetramethylpyrazine on early afterdepolarizations induced by calcium paradox injury in myocardium of rats ex vivo," Chinese Journal of Clinical Rehabilitation, vol. 10, no. 39, pp. 104-106, 2006.

[43] K. Shiroto, H. Otani, F. Yamamoto, C. Huang, N. Maulik, and D. K. Das, "MK2 $2^{-/-}$gene knockout mouse hearts carry anti-apoptotic signal and are resistant to ischemia reperfusion injury," Journal of Molecular and Cellular Cardiology, vol. 38, no. 1, pp. 93-97, 2005.

[44] K. Y. Kwon, C. H. Cho, S. P. Kim, C. K. Park, and S. H. Jheon, "Apoptosis induced by preservation and reperfusion in canine lung transplantation," Transplantation Proceedings, vol. 35, no. 1, pp. 134-137, 2003.

[45] H. Duan, Y. Li, S. H. Zhang et al., "Effects of ligustrazine on apoptosis in rats with myocardial ischemia reper fusion injury," Chinese Journal of Cardiology, vol. 27, no. 4, pp. 294-296, 1999.

[46] D. J. Xie, R. H. Chen, H. L. Ren et al., "Interference of myocardial apoptosis in pressure overload SD rats with tetramethylpyrazine," Chinese Heart Journal, vol. 16, no. 1, pp. 26-28, 2004.

[47] J. Wan, D. Ye, P. Wu, L. Zhang, X. Gong, and Y. Huang, "Effect of tetramethylpyrazine on lipopolysaccharides induced macrophage cyclo-oxidase-2 expression and apoptosis of cardiac myocytes," Chinese Journal of Integrated Traditional and Western Medicine, vol. 24, no. 10, pp. 906-911, 2004.
[48] J. Liu and P. W. Niu, "Protective effects of chuan xiong qin against ischemia-reperfusion injury in rats," China Practical Medicine, vol. 6, no. 2, pp. 29-30, 2011.

[49] X. Cheng, L. Zhang, J. Hu, L. Sun, and G. Du, "Neuroprotective effects of tetramethylpyrazine on hydrogen peroxide-induced apoptosis in PC12 cells," Cell Biology International, vol. 31, no. 5, pp. 438-443, 2007.

[50] G. M. Zhang, W. F. Wang, X. P. Wang et al., "The myocardial apoptosis and the expression of Fas and FasL genes in ischemic myocardium of rats," Progress of Anatomical Sciences, vol. 15, no. 1, pp. 100-102, 2009.

[51] J. Zhang, L. Q. Liu, L. H. Yu et al., "Effects of ligustrazine on the apoptosis in rats with myocardial ischemia reperfusion injury," Acta Academiae Medicine Jiangxi, vol. 47, no. 6, pp. 20-22, 2007.

[52] Z. Y. Zhai, J. H. Yang, S. T. Zhang et al., "Role of Janus kinase $2 /$ signal transducer and activator of transcription 3 signaling pathway in attenuation of myocardial ischemia-reperfusion injury by teramethylpyrazine in rats," Chinese Journal of Anesthesiology, vol. 31, no. 8, pp. 1005-1008, 2011.

[53] R. Y. Zhao, W. Hao, X. J. Meng et al., "Effects of ligustrazine ferulate postconditioning on myocardial ischemia reperfusion injury in rats," Journal of China Medical University, vol. 41, no. 11, pp. 1018-1021, 2012.

[54] N. G. Frangogiannis, C. W. Smith, and M. L. Entman, “The inflammatory response in myocardial infarction," Cardiovascular Research, vol. 53, no. 1, pp. 31-47, 2002.

[55] S.-L. Liao, T.-K. Kao, W.-Y. Chen et al., "Tetramethylpyrazine reduces ischemic brain injury in rats," Neuroscience Letters, vol. 372, no. 1-2, pp. 40-45, 2004.

[56] L. Zhang, "Research progress on mechanism of of prevention and treatment of Chinese medicinal herb on myocardial ischemia reperfusion injury," Liaoning Journal of Traditional Chinese Medicine, vol. 37, no. 10, pp. 2070-2072, 2010.

[57] M. F. Hu, L. Z. Shang, and D. W. Wei, "Protective effects of ligustrazine on myocardial ischemia reperfusion injury in rats," Shanghai Journal of Traditional Chinese Medicine, vol. 42, no. 4, pp. 66-68, 2008.

[58] M. P. Castaneda, A. Swiatecka-Urban, M. M. Mitsnefes et al., "Activation of mitochondrial apoptotic pathways in human renal allografts after ischemia-reperfusion injury," Transplantation, vol. 76, no. 1, pp. 50-54, 2003.

[59] L. Z. Shang, F. Wang, and J. R. Wang, "The effects of ligustrazine on expression of $\mathrm{p} 38 \mathrm{MAPK}$ and TNF- $\alpha$ in rats with myocardial ischemia reperfusion injury," Journal of Henan University of Chinese Medicine, vol. 23, no. 5, pp. 24-25, 2008.

[60] Z. H. Pan and X. Jin, "Protective effects of combination of ligustrazine and ferulic acid on myocardial injury induced by ischemia and reperfusion in rats," Tianjin Medical Journal, vol. 36, no. 10, pp. 796-798, 2008.

[61] J. R. Yang, M. X. Zhang, L. T. Chang et al., "Protective effects of ligustrazine,ferulic acid and their combination on myocardial injury induced by ischemia-reperfusion in rats," Chinese Traditional and Herbal Drugs, vol. 39, no. 7, pp. 10541056, 2008.

[62] Y. Wang, S. H. Mi, S. J. Jia et al., "Research progress in drug treatment of myocardial ischemia reperfusion injury," Chinese Journal of Practical Internal Medicine, vol. 32, no. 12, pp. 958960, 2012.

[63] D. Fulton, J. P. Gratton, T. J. Mccabe et al., "Regulation of endothelium-derived nitric oxide production by the protein kinase Akt," Nature, vol. 399, no. 6736, pp. 597-601, 1999. 
[64] L. Lv, S. Jiang, J. Xu, J. Gong, and Y. Cheng, "Protective effect of ligustrazine against myocardial ischaemia reperfusion in rats: the role of endothelial nitric oxide synthase," Clinical and Experimental Pharmacology and Physiology, vol. 39, no. 1, pp. 20-27, 2012.

[65] R. X. Liang, F. L. Liao, and D. Han, "Study of mechanism by ligustrazine preconditioning protection on myocardial ischemia and reperfussion injury," Pharmacology and Clinics of Chinese Material Medica, vol. 17, no. 3, pp. 8-10, 2001.

[66] H. P. Chen, M. He, Q. R. Huang, G. Zeng, and D. Liu, "Delayed protection of tetramethylpyrazine on neonatal rat cardiomyocytes subjected to anoxia-reoxygenation injury," Basic and Clinical Pharmacology and Toxicology, vol. 100, no. 6, pp. 366371, 2007.

[67] C. Q. Li and Y. X. Li, "Protective effects be related with adipeincton ligustrzaine on myocardial ischemia reperfusion injury," Guide of China Medicine, vol. 8, no. 36, pp. 215-216, 2010.

[68] W. T. Wang, Z. J. Xu, and L. N. Lin, "Effect of ligustrazine on nitric oxide and endothelin in the reperfusion injury after myocardial ischemia in rabbits," Chinese Journal of Pathopsiology, vol. 17, no. 3, pp. 230-234, 2001.

[69] R. X. Liang, T. Z. Guan, F. L. Liao et al., "Ligustrazine preconditioning protects endothelium from myocardial ischemia and reperfussion injury in rats," Chinese Journal of Microcirculation, vol. 12, no. 4, pp. 4-5, 2002.

[70] M. Li, M. Q. Zhao, S. S. K. Kumar Durairajan et al., "Protective effect of tetramethylpyrazine and salvianolic acid B on apoptosis of rat cerebral microvascular endothelial cell under high shear stress," Clinical Hemorheology and Microcirculation, vol. 38, no. 3, pp. 177-187, 2008.

[71] B. Shu, C. J. Zhou, Y. H. Ma et al., "Progress in studies on pharmacological effect of components rhizoma chuanxiong," Chinese Pharmacologieal Bulletin, vol. 22, no. 9, pp. 1043-1045, 2006.

[72] C. Y. Guan, "Application status in the field of cardiovascular effects of ligustrazine andexisting problems," Chinese Journal of Integrated Traditional and Western Medicine, vol. 23, no. 5, p. 378, 2003.

[73] L. Han and C. Ke-ji, "Study on the protective effect of ligustrazine on myocardial ischemia reperfusion injury," Pharmaceutical Journal of Chinese People's Liberation Army, vol. 18, no. 1, pp. 41-43, 2002. 


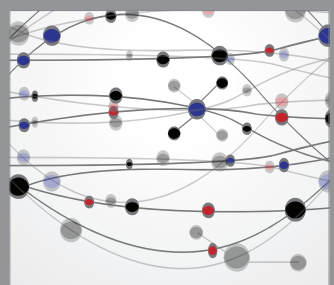

The Scientific World Journal
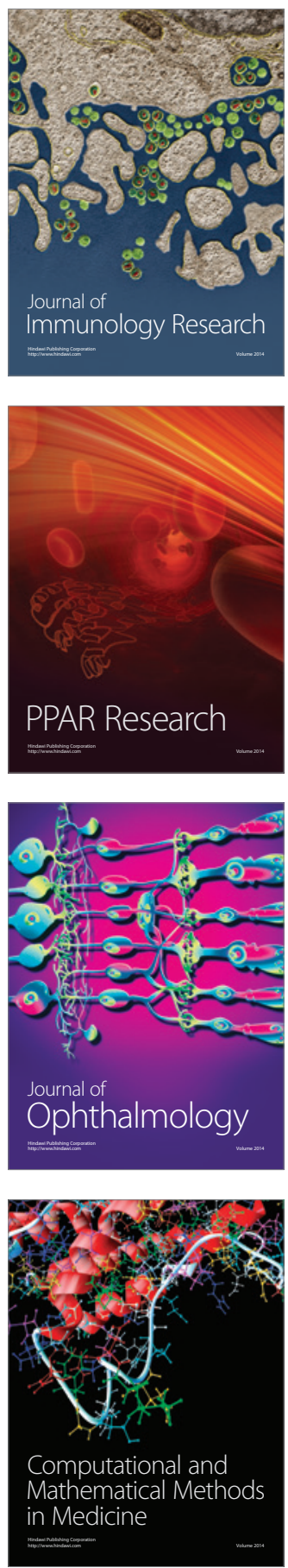

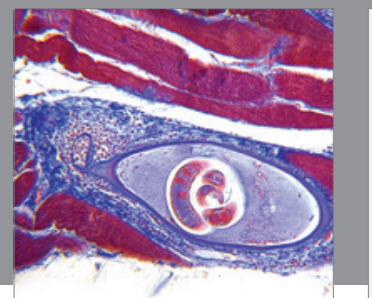

Gastroenterology

Research and Practice
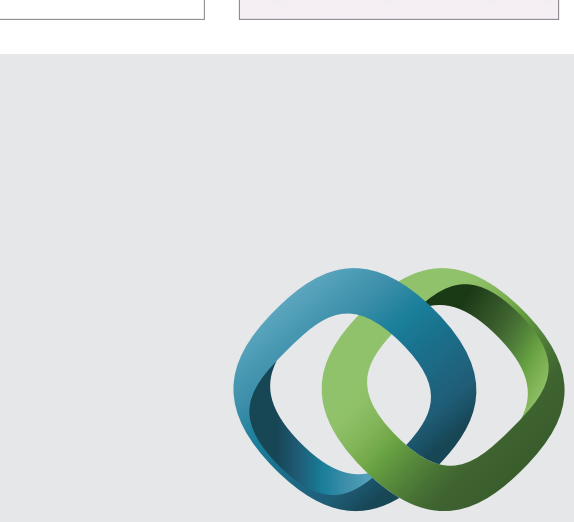

\section{Hindawi}

Submit your manuscripts at

http://www.hindawi.com
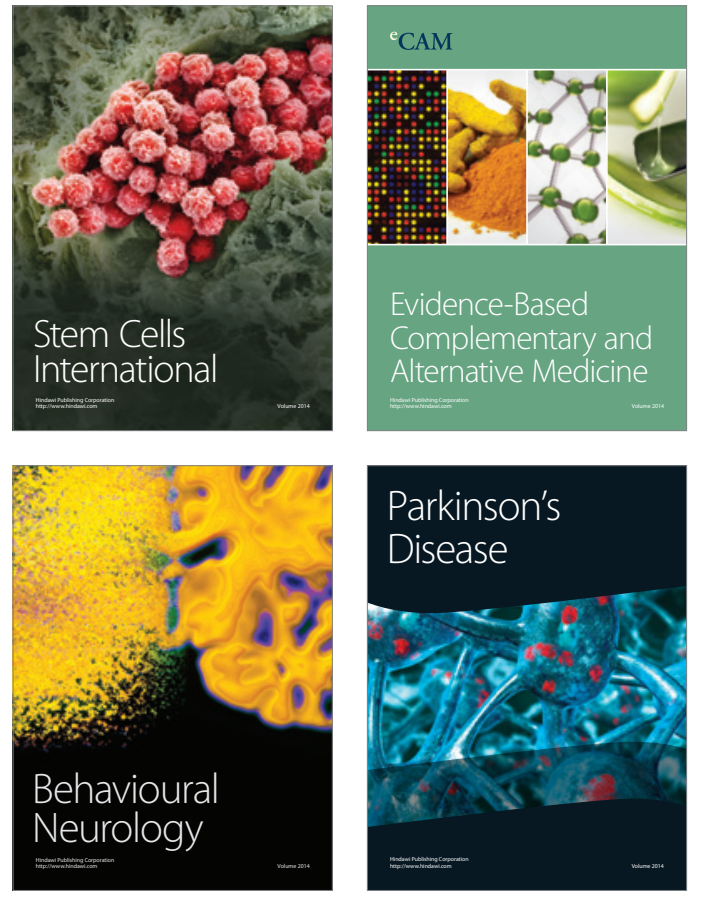
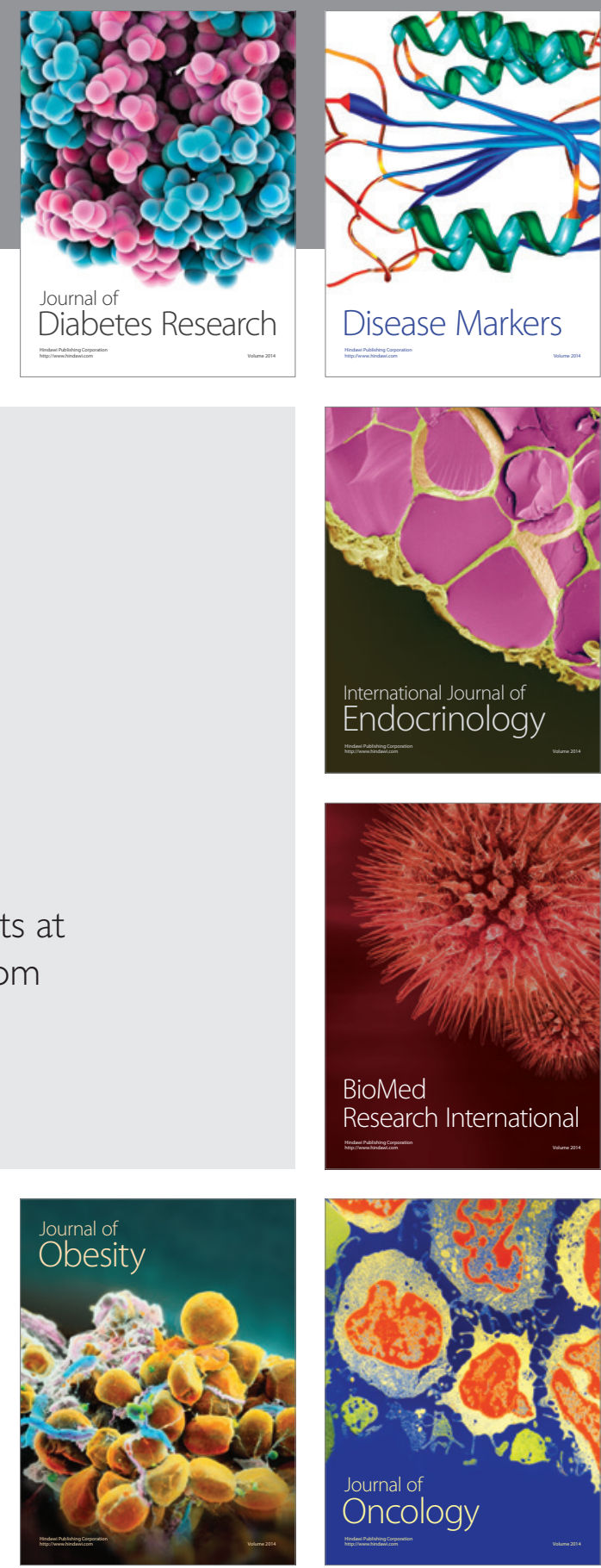

Disease Markers
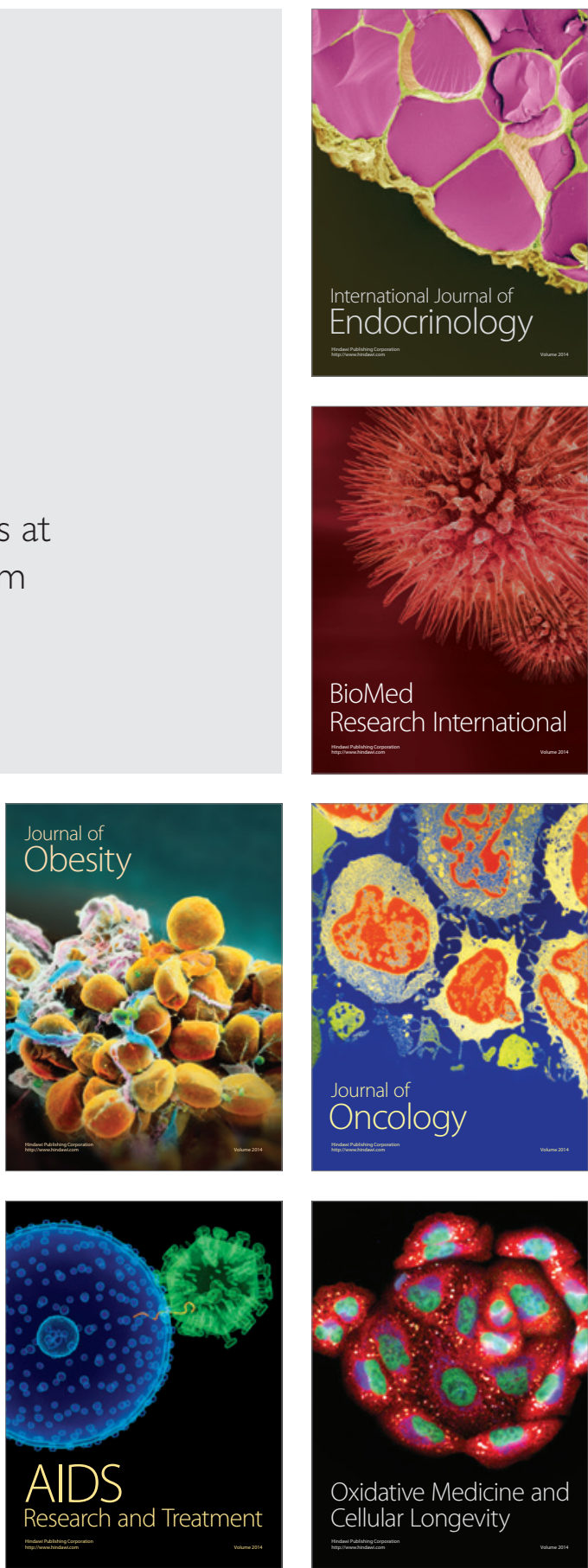